\title{
The Capacity of Three-Dimensional Wireless Ad Hoc Networks
}

\author{
Pan $\mathrm{Li}^{*}$, Miao Pan ${ }^{\dagger}$, and Yuguang Fang ${ }^{\dagger}$ \\ *Department of Electrical and Computer Engineering, Mississippi State University, MS 39762 \\ ${ }^{\dagger}$ Department of Electrical and Computer Engineering, University of Florida, FL 32611 \\ Email: li@ece.msstate.edu, miaopan@ufl.edu,fang@ece.ufl.edu
}

\begin{abstract}
Network capacity investigation has been intensive in the past few years. A large body of work has appeared in the literature. However, so far most of the effort has been made on two-dimensional wireless networks only. With the great development of wireless technologies, wireless networks are envisioned to extend from two-dimensional space to threedimensional space. In this paper, we investigate for the first time the throughput capacity of $3 \mathrm{D}$ regular ad hoc networks (RANETs) and of 3D heterogeneous ad hoc networks (HANETs), respectively, by employing a generalized physical model. In 3D RANETs, we assume that the nodes are regularly placed, while in 3D HANETs, we consider that the nodes are distributed according to a general Nonhomogeneous Poisson Process (NPP). We find both lower and upper bounds in both types of networks in a broad power propagation regime, i.e., when the path loss exponent is no less than 2 .
\end{abstract}

\section{INTRODUCTION}

Network capacity investigation has been intensive in the past few years. A big chunk of work exploring the capacity of wireless networks has appeared in the literature. When we ask ourselves why we should engage in this pursuit, two reasons should be obvious. First, network capacity is the asymptotic property of network performance. In face of the emerging large-scale networks of a large number of connected objects, asymptotic capacity is no longer a cliché and becomes even more critical. Second, network capacity predicts network performance in case of different paradigms as a function of the number of nodes in the network, regardless of detailed protocol design. In contrast, as an alternative way to evaluate the network performance, simulation or numerical results can only be obtained for a certain number of nodes and are hence deterministic. Besides, these results are only available after designing all the protocols considering every detail, and may also require a lot of computing resource and time for a large network. Therefore, capacity investigation is interesting and important in wireless networks. However, it is also a very challenging task.

Gupta and Kumar [11] initiate the study on the capacity of wireless networks and show that the per-node throughput capacity (with unit bits per second) is $\Theta(1 / \sqrt{n \log n})$ in random ad hoc networks and the per-node transport capacity (with unit bit-meters per second) is $\Theta(1 / \sqrt{n})$ in arbitrary ad

This work was partially supported by the U.S. National Science Foundation under grants CNS-0916391 and CNS-0721744. The work of Y. Fang was also partially supported by the National Natural Science Foundation of China under grant 61003300 and China 111 Project under grant B08038. hoc networks, where $n$ is the number of nodes in the network. A large body of work (e.g., [1], [2], [4], [6]-[8], [16], [22][24]) continues to study the capacity of static ad hoc networks with different network settings, while a tremendous amount of effort (e.g., [3], [10], [13], [17], [20], [28]) is also made on the capacity of mobile ad hoc networks, showing that mobility can significantly improve network capacity. The bulk of work on the capacity of hybrid wireless networks, such as [15], [18], [19], [21], [29], [30], proposes to place base stations in wireless networks and finds that network capacity can also be boosted.

However, all the aforementioned work is conducted on twodimensional networks only. With the great development of wireless technologies, wireless networks are envisioned to extend from two-dimensional space to three-dimensional space, connecting all kinds of objects such as computers, sensors, actuators, mobile phones, TVs, refrigerators, washers/driers, smart power meters, clothes, food, medicines, and cars. The future three-dimensional (3D) wireless networks will be a fusion of the digital world and the physical world and bring together everything from individuals to objects, from data to services, etc. Only a couple of papers like [12] tentatively study the capacity of 3D wireless networks. In particular, [12] explores the transport capacity in 3D arbitrary ad hoc networks and the throughput capacity in 3D random ad hoc networks, using both Protocol Model and Physical Model. In this paper, we investigate for the first time the throughput capacity of $3 \mathrm{D}$ regular ad hoc networks and of 3D heterogeneous ad hoc networks, respectively, by employing a generalized physical model.

More specifically, we consider a network with $n$ nodes distributed in a three-dimensional cube with edge $L$, where $L=n^{\alpha / 3}(0 \leq \alpha \leq 1)$, and the network volume $|\mathcal{V}|=L^{3}$. In $3 \mathrm{D}$ regular ad hoc networks (RANETs), assuming that the $n$ nodes are regularly placed, we find that the throughput capacity is lower bounded by $n^{(\gamma-4) / 3}$ when $2 \leq \gamma<3$, by $n^{-\frac{1}{3}} / \ln n$ when $\gamma=3$, and by $n^{-\frac{1}{3}}$ when $\gamma>3$, where $\gamma$ is the path loss exponent, and is upper bounded by $\frac{P_{\max }}{P_{\min }} n^{-\frac{1}{3}}$ when the transmission power of the nodes can be tuned between $P_{\min }$ and $P_{\max }$ with $0<P_{\min } \leq P_{\max }$. In $3 \mathrm{D}$ heterogeneous ad hoc networks (HANETs), we assume that the $n$ nodes are distributed according to a general Nonhomogeneous Poisson Process (NPP), with the local intensity at point $\xi$ in the network denoted by $\Psi(\xi)$, and $\int_{\mathcal{V}} \Psi(\xi) \mathrm{d} \xi=n$. 
The minimum and the maximum of $\Psi(\xi)$ are denoted by $\underline{\Psi}$ and $\bar{\Psi}$, respectively, which both scale with $n$. We show that the throughput capacity is lower bounded by $\frac{\underline{\Psi}^{2}}{\bar{\Psi}^{2} \ln n}\left(\frac{\ln n}{\underline{\underline{y}} n^{\alpha}}\right)^{\frac{4-\gamma}{3}}$ when $2 \leq \gamma<3$, by $\frac{\underline{\Psi}^{2}}{\bar{\Psi}^{2}(\ln n) \ln \left(\frac{\Psi n^{\alpha}}{\ln n}\right)}\left(\frac{\ln n}{\underline{\Psi} n^{\alpha}}\right)^{\frac{1}{3}}$ when $\gamma=3$, and by $\frac{\Psi^{2}}{\bar{\Psi}^{2} \ln n}\left(\frac{\ln n}{\underline{\Psi} n^{\alpha}}\right)^{\frac{1}{3}}$ when $\gamma>3$, and is upper bounded by $\min \left\{\frac{P_{\max }}{P_{\min }}, \frac{P_{\max }}{P_{\min }}\left(\bar{\Psi}^{2} n^{2 \alpha-3}\right)^{\frac{1}{3}}\right\}$ when the nodes' transmission powers fall into the interval $\left[P_{\min }, P_{\max }\right]$.

The rest of this paper is organized as follows. In Section II we introduce some definitions and models we will use throughout this paper. Section III and Section IV present the capacity of 3D regular ad hoc networks and of 3D heterogeneous ad hoc networks, respectively. We finally conclude this paper in Section V.

\section{DEFINITIONS AND MODELS}

In this section, we introduce the definitions and models we will use in this study.

\section{A. Definitions}

Throughput: As defined in the usual way, the time average of the number of bits per second that can be transmitted by each node to its destination is called the per-node throughput. The sum of per-node throughput over all the nodes in a network is called the throughput of the network.

Feasible Throughput: We say that a per-node throughput, denoted by $\lambda(n)$, is feasible if there exists a spatial and temporal scheduling scheme that yields a per-node throughput of $\lambda(n)$ bits per second. Let $\lambda_{i}(n)$ denote the throughput of node $i$. We say that a per-node throughput, denoted by $\lambda(n)$, is feasible by all nodes if there exists a spatial and temporal scheduling scheme such that $\lambda_{i}(n) \geq \lambda(n)$ for all $i \in[1, n]$, and is feasible on average if there exists a spatial and temporal scheduling scheme such that $\frac{1}{n} \sum_{i=1}^{n} \lambda_{i}(n) \geq \lambda(n)$. In this paper, we will derive a per-node throughput feasible on average unless otherwise specified, which we call "per-node throughput" for simplicity.

Per-node Throughput Capacity: We say that the per-node throughput capacity in the network is of order $\mathrm{O}(f(n))$ bits/sec if there is a deterministic constant $0<c_{1}<+\infty$ such that

$$
\liminf _{n \rightarrow+\infty} \mathrm{P}\left(\lambda(n)=c_{1} f(n) \text { is feasible }\right)<1,
$$

and is of order $\Theta(f(n))$ bits/sec if there are deterministic constants $0<c_{2}<c_{3}<+\infty$ such that

$$
\begin{aligned}
& \liminf _{n \rightarrow+\infty} \mathrm{P}\left(\lambda(n)=c_{2} f(n) \text { is feasible }\right)=1, \\
& \liminf _{n \rightarrow+\infty} \mathrm{P}\left(\lambda(n)=c_{3} f(n) \text { is feasible }\right)<1 .
\end{aligned}
$$

\section{B. Network Model}

We consider a network with $n$ nodes distributed in a threedimensional cube with edge $L$, where $L=n^{\alpha / 3}(0 \leq \alpha \leq$ 1 ), and the network volume $|\mathcal{A}|=L^{3}$. Thus, we can model all kinds of networks including dense networks $(\alpha=0)$ in [19], extended networks $(\alpha=1)$ in [15], and semi-extended networks $(0<\alpha<1)$ in [7]. We also assume that the network nodes can have transmission powers ranging from $P_{\min }$ to $P_{\max }$ with $0<P_{\min } \leq P_{\max }$.

3D Regular Ad Hoc Networks (RANETs): We first assume that the $n$ nodes are regularly placed in the network. An example is shown in Fig. 1(a), in which there is one node at the center of each cubelet.

3D Heterogeneous Ad Hoc Networks (HANETs): We then extend our study to the case in which the $n$ nodes are distributed according to a general Nonhomogeneous Poisson Process (NPP), with the local intensity at point $\xi$ in the network denoted by $\Psi(\xi)$, and $\int_{\mathcal{A}} \Psi(\xi) \mathrm{d} \xi=n$. The minimum and the maximum of $\Psi(\xi)$ are denoted by $\underline{\Psi}$ and $\bar{\Psi}$, respectively, which both scale with $n$. We also assume $\underline{\Psi} n^{\alpha}=\omega(\ln n)$. Fig. 1(b) shows one example of 3D HANETs.

Moreover, we follow the process in [10] to choose random sender-receiver pairs so that each node is a source node for one flow and a destination node for at most $\mathrm{O}(1)$ flows.

\section{Channel Capacity Model}

Let $d_{i j}$ denote the distance between a node $i$ and another node $j$. The reception power at node $j$ of the signal from node $i$, denoted by $P_{i j}$, follows the power propagation model described in [25], i.e.,

$$
P_{i j}=C \frac{P_{i}}{d_{i j}^{\gamma}},
$$

where $P_{i}$ is the transmission power of node $i, \gamma$ is the path loss exponent, and $C$ is a constant related to the antenna profiles of the transmitter and the receiver, wavelength, and so on. Note that $\gamma \geq 2$.

We consider the Shannon Capacity as the channel capacity between two nodes. Specifically, a transmission from node $i$ to node $j$ can have channel capacity, $R_{i j}$, which is calculated as follows:

$$
R_{i j}=B \log _{2}\left(1+S I N R_{i j}\right)
$$

where $B$ is the channel bandwidth, and $S I N R_{i j}$ is the SINR (Signal-to-Interference plus Noise Ratio) of the signal from node $i$ to node $j$. In this study, we consider the channel bandwidth $B$ to be a constant.

\section{CApacity of Three-Dimensional Regular AD HOC NETWORKS}

In this section, we investigate the capacity of threedimensional regular ad hoc networks (RANETs). Both a lower bound and an upper bound on the capacity will be presented, respectively.

\section{A. A Lower Bound on Capacity}

We first find a lower bound on capacity by obtaining an achievable throughput. As mentioned in Section II-B, a 3D RANET can be divided into cubelets with an edge length of $l=n^{(\alpha-1) / 3}$ and a node at the center. We let all nodes employ the same transmission power so that the transmission range is $l$, the distance between two neighboring nodes.

We divide a 3D RANET into groups each of which contains twenty-seven cubelets, as shown in Fig. 1(a). The twenty-seven 


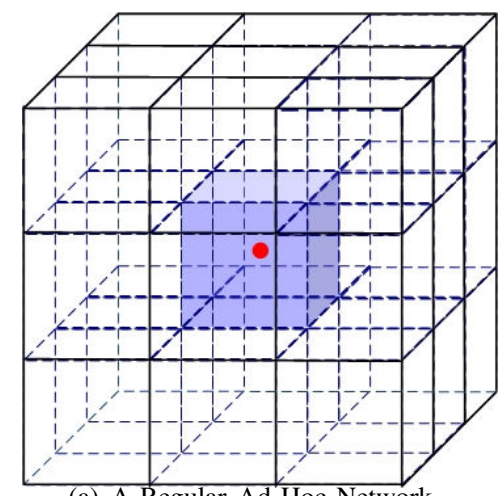

(a) A Regular Ad Hoc Network.

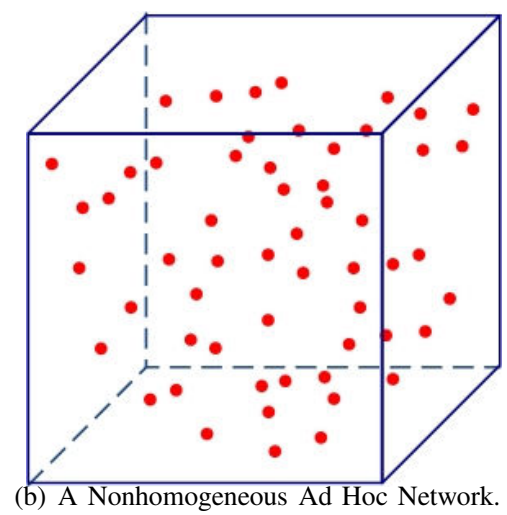

Fig. 1. Examples of 3D ad hoc networks. cubelets in each group are numbered from 1 to 27 in the same way. We further divide time into sequences of successive slots, denoted by $t(t=0,1,2,3, \ldots)$. During a time slot $t$, all nodes in cubelets that are numbered $(t \bmod 27)+1$ are allowed to transmit packets.

Consider a time slot when the node $T_{i}$ in cubelet $C_{i}$ is allowed to transmit to another node $R_{i}$. Denote by $P_{t}$ the nodes' transmission power. The reception power level at $R_{i}$, denoted by $P_{r, i}$, is thus

$$
P_{r, i}=\frac{C P_{t}}{l^{\gamma}} .
$$

Besides, those nodes that may interfere with the transmission of $T_{i}$ are located on the sides of concentric cubelets centered at $C_{i}$. At the $j$ th tier, the number of interfering nodes, denoted by $N_{j}$, is

$$
\begin{aligned}
N_{j} & =(2 j+1)^{3}-(2 j-1)^{3} \\
& =2 \times\left(12 j^{2}+1\right) \\
& \leq 26 j^{2}
\end{aligned}
$$

and the distance from an interfering transmitter to $R_{i}$, denoted by $l_{j}$, satisfies

$$
l_{j} \geq(3 j-1) \cdot l .
$$

Thus, according to the power propagation model in (1), the cumulative interference at $R_{i}$, denoted by $I_{i}$, can be calculated as follows:

$$
\begin{aligned}
I_{i} & \leq \sum_{j=1}^{N_{\max }} 26 j^{2} \times \frac{C P_{t}}{[(3 j-1) l]^{\gamma}} \\
& \leq \frac{26 C P_{t}}{l^{\gamma}}\left[1+\sum_{j=2}^{N_{\max }}(3 j-1)^{2-\gamma}\right] \\
& =\frac{26 C P_{t}}{l^{\gamma}}\left[1+\sum_{j=1}^{N_{\max }-1}(3 j+2)^{2-\gamma}\right]
\end{aligned}
$$

where $N_{\max }$ is the maximum number of tiers. Obviously, we have $N_{\max }=n^{\frac{\alpha}{3}} / n^{\frac{\alpha-1}{3}}=n^{\frac{1}{3}}$.

Case I: $\gamma=2$.
When $\gamma=2$, the cumulative interference is

$$
\begin{aligned}
I_{i} & \leq \frac{26 C P_{t}}{l^{\gamma}}\left[1+\sum_{j=1}^{N_{\max }-1} 1\right] \\
& =\frac{26 C P_{t}}{l^{\gamma}} \cdot n^{\frac{1}{3}} .
\end{aligned}
$$

Like in [14] [27], we consider an interference dominated environment where noise can be ignored. Thus, the SINR at the receiver $R_{i}$, denoted by $S I N R_{i}$, is

$$
S I N R_{i} \geq \frac{\frac{C P_{t}}{l \gamma}}{\frac{26 C P_{t}}{l^{\gamma}} \cdot n^{\frac{1}{3}}}=\frac{1}{26} n^{-\frac{1}{3}} .
$$

According to the Shannon Capacity, the transmission rate from $T_{i}$ to $R_{i}$, denoted by $R_{C}^{i}$, is

$$
R_{C}^{i} \geq B \log _{2}\left(1+\frac{1}{26} n^{-\frac{1}{3}}\right) \approx \frac{B}{26} n^{-\frac{1}{3}} .
$$

Case II: $2<\gamma<3$.

In this case, we get

$$
\begin{aligned}
I_{i} & \leq \frac{26 C P_{t}}{l^{\gamma}}\left[1+\int_{0}^{N_{\max }}(3 j+2)^{2-\gamma} \mathbf{d} j\right] \\
& =\frac{26 C P_{t}}{l^{\gamma}}\left[1+\left.\frac{(3 j+2)^{3-\gamma}}{3(3-\gamma)}\right|_{0} ^{N_{\max }}\right] \\
& \leq \frac{26 C P_{t}}{l^{\gamma}}\left[1+\frac{\left(4 N_{\max }\right)^{3-\gamma}}{3(3-\gamma)}\right] \\
& \leq \frac{52 \cdot 4^{3-\gamma}}{3(3-\gamma)} \cdot \frac{C P_{t} N_{\max }^{3-\gamma}}{l^{\gamma}}
\end{aligned}
$$

and hence

$$
S I N R_{i} \geq \frac{\frac{C P_{t}}{l \gamma}}{\frac{52 \cdot 4^{3-\gamma}}{3(3-\gamma)} \cdot \frac{C P_{t} N_{\max }^{3-\gamma}}{l^{\gamma}}}=\frac{3(3-\gamma)}{52 \cdot 4^{3-\gamma}} n^{\frac{1}{3}(\gamma-3)} .
$$

Since $\gamma<3$, we can obtain that

$$
\begin{aligned}
R_{C}^{i} & \geq B \log _{2}\left(1+\frac{3(3-\gamma)}{52 \cdot 4^{3-\gamma}} n^{\frac{1}{3}(\gamma-3)}\right) \\
& \approx \frac{3(3-\gamma) B}{52 \cdot 4^{3-\gamma}} n^{\frac{1}{3}(\gamma-3)} .
\end{aligned}
$$

Case III: $\gamma=3$. 
When $\gamma=3$, we can get that

$$
\begin{aligned}
I_{i} & \leq \frac{26 C P_{t}}{l^{\gamma}}\left[1+\int_{0}^{N_{\max }} \frac{1}{3 j+2} \mathbf{d} j\right] \\
& =\frac{26 C P_{t}}{l^{\gamma}}\left[1+\frac{1}{3} \ln \left(3 N_{\max }+2\right)-\frac{1}{3} \ln 2\right] \\
& \leq \frac{26 C P_{t}}{l^{\gamma}}\left[\frac{1}{3} \ln \left(4 N_{\max }\right)+1-\frac{1}{3} \ln 2\right] \\
& =\frac{26 C P_{t}}{l^{\gamma}}\left[\frac{1}{3} \ln N_{\max }+1+\frac{1}{3} \ln 2\right] \\
& \leq \frac{26 C P_{t}}{l^{\gamma}} \ln N_{\max } .
\end{aligned}
$$

As a result, the SINR of the transmission from $T_{i}$ to $R_{i}$ is

$$
S I N R_{i} \geq \frac{\frac{C P_{t}}{l \gamma}}{\frac{26 C P_{t}}{l \gamma} \ln N_{\max }}=\frac{3}{26 \ln n},
$$

and

$$
R_{C}^{i} \geq B \log _{2}\left(1+\frac{3}{26 \ln n}\right) \approx \frac{3 B}{26 \ln n} .
$$

Case IV: $\gamma>3$.

In this case, the cumulative interference can be calculated as

$$
\begin{aligned}
I_{i} & \leq \frac{26 C P_{t}}{l^{\gamma}}\left[1+\int_{0}^{N_{\max }}(3 j+2)^{2-\gamma} \mathbf{d} j\right] \\
& =\frac{26 C P_{t}}{l^{\gamma}}\left[1+\frac{2^{3-\gamma}}{3(\gamma-3)}\right] \\
& <\frac{26 C P_{t}}{l^{\gamma}}\left[1+\frac{1}{3(\gamma-3)}\right] \\
& =\frac{26 C P_{t}}{l^{\gamma}} \cdot \frac{3 \gamma-8}{3 \gamma-9} .
\end{aligned}
$$

Thus, we can have that

$$
S I N R_{i} \geq \frac{\frac{C P_{t}}{l \gamma}}{\frac{26 C P_{t}}{l \gamma} \cdot \frac{3 \gamma-8}{3 \gamma-9}}=\frac{3 \gamma-9}{26(3 \gamma-8)}
$$

and

$$
R_{C}^{i} \geq B \log _{2}\left(1+\frac{3 \gamma-9}{26(3 \gamma-8)}\right)
$$

which can be lower bounded by a constant.

Note that every node transmits every 27 time slots. The final results follow subsequently.

Lemma 1: In 3D RANETs, the transmission rate of each node, denoted by $R_{C}$, is

$$
R_{C} \geq \begin{cases}\frac{B}{c_{1}} n^{-\frac{1}{3}} & \text { if } \gamma=2 \\ \frac{B}{c_{2}} n^{\frac{1}{3}(\gamma-3)} & \text { if } 2<\gamma<3 \\ \frac{B}{c_{3} \ln n} & \text { if } \gamma=3 \\ \frac{B}{c_{4}} & \text { if } \gamma>3\end{cases}
$$

where $c_{1}=702, c_{2}=468 \cdot 4^{3-\gamma} /(3-\gamma), c_{3}=234, c_{4}=$ $27 \log _{2}^{-1}\left(1+\frac{3 \gamma-9}{26(3 \gamma-8)}\right)$.

Besides, we use the following routing strategy to relay the packets. Specifically, as shown in Fig. 2, assume that a source node $S$ is located at $\left(x_{s}, y_{s}, z_{s}\right)$ and its destination node $D$ is

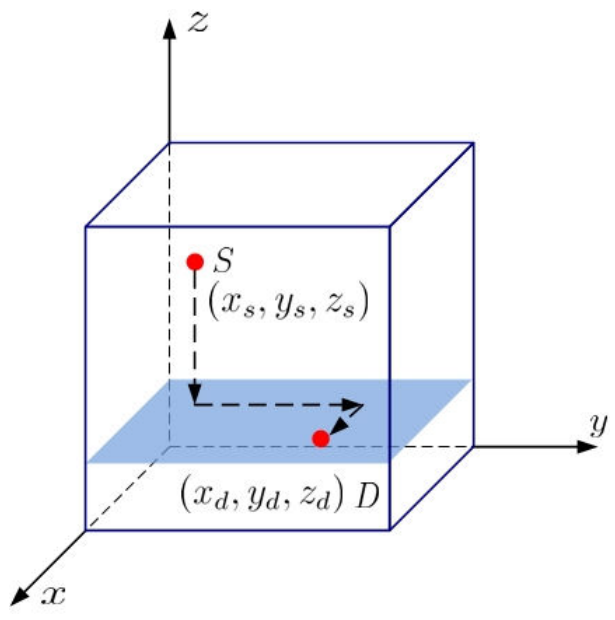

Fig. 2. The routing strategy used for ad hoc mode transmissions.

located at $\left(x_{d}, y_{d}, z_{d}\right)$. Packets from this source node are firstly relayed from $\left(x_{s}, y_{s}, z_{s}\right)$ to $\left(x_{s}, y_{s}, z_{d}\right)$, then to $\left(x_{s}, y_{d}, z_{d}\right)$, and finally to $\left(x_{d}, y_{d}, z_{d}\right)$.

Denote the average distance between a source-destination pair by $\bar{L}$. We can get that [5]

$$
\bar{L} \leq \frac{1}{\sqrt{6}}\left(\frac{1+2 \sqrt{1-1 / 5}}{3}\right)^{1 / 2} \cdot \sqrt{3} n^{\alpha / 3} \approx 0.68 n^{\alpha / 3} .
$$

Let $c_{5}=0.68$. Then, $\bar{L} \leq c_{5} n^{\alpha / 3}$ and the average number of hops, denoted by $\bar{H}$, is

$$
\begin{aligned}
\bar{H} & =\frac{\left\|z_{s}-z_{d}\right\|}{l}+\frac{\left\|y_{s}-y_{d}\right\|}{l}+\frac{\left\|x_{s}-x_{d}\right\|}{l} \\
& \leq \frac{3 \bar{L}}{l} .
\end{aligned}
$$

Thus, a per-node throughput, denoted by $\lambda(n)$, is feasible if

$$
\lambda(n) \cdot \bar{H} \leq R_{C}
$$

i.e.,

$$
\lambda(n) \leq \frac{1}{3 c_{5} n^{1 / 3}} R_{C},
$$

which leads to the following theorem.

Theorem 1: A lower bound on the capacity of 3D RANETs is

$$
\lambda(n)=\Omega \begin{cases}\frac{B}{3 c_{1} c_{5}} n^{-\frac{2}{3}} & \text { if } \gamma=2, \\ \frac{B}{3 c_{2} c_{5}} n^{\frac{\gamma-4}{3}} & \text { if } 2<\gamma<3, \\ \frac{B}{3 c_{3} c_{5} \ln n} n^{-\frac{1}{3}} & \text { if } \gamma=3, \\ \frac{B}{3 c_{4} c_{5}} n^{-\frac{1}{3}} & \text { if } \gamma>3 .\end{cases}
$$

\section{B. An Upper Bound on Capacity}

Next, we investigate the upper bound on the network capacity by finding the maximum amount of information that passes a cut plane in a 3D RANET.

As shown in Fig. 3, a cut plane is chosen such that the network space is divided into two parts each of which contains $n / 2$ nodes. The transmitters are on the left side and the receivers are on the right side. We divide the left part of the 


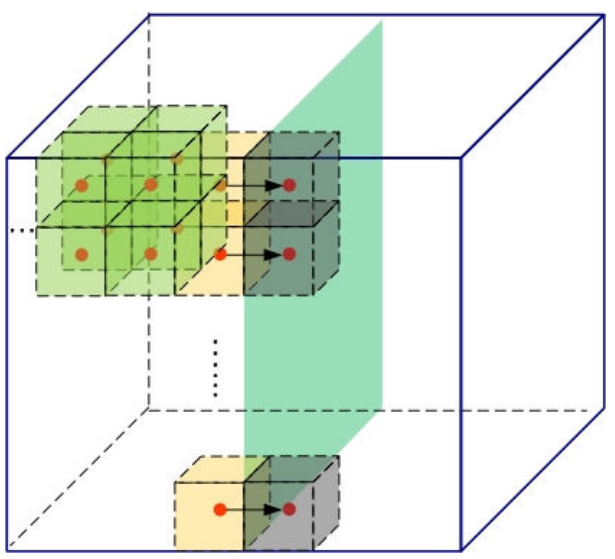

Fig. 3. Finding an upper bound on capacity using a cut plane.

network into tiers each of which are composed of blocks of different sizes. Specifically, at tier $j(j \geq 1)$, the blocks have an edge length of $2^{j-1} \cdot l$. We now show that each block as a whole can have at most a constant transmission rate.

Recall that the transmission power of each node falls into the interval $\left[P_{\min }, P_{\max }\right]$ with $P_{\min } \leq P_{\max }$. We use $P_{r}^{j}$, $I^{j}$, and $S I N R^{j}$ to denote the reception power, interference suffered at receiver, and the SINR of transmissions originated from transmitters in tier $j$ blocks, respectively. We also denote the total transmission rate of all nodes in a tier $j$ block by $R_{C}^{j}$. Then, at tier 1 , since the minimum distance between a transmitter and a receiver is $l$, we have

$$
P_{r}^{1} \leq \frac{P_{\max }}{l^{\gamma}}
$$

Besides, the minimum interference is observed when there is only one other transmitter right next to the current one with the minimum transmission power $P_{\min }$. Thus, we can get

$$
I^{1} \geq \frac{P_{\min }}{(\sqrt{2} l)^{\gamma}}
$$

As a result, neglecting the noise, we can have

$$
S I N R^{1} \leq 2^{\frac{\gamma}{2}} \frac{P_{\max }}{P_{\min }}
$$

and hence

$$
R_{C}^{1} \leq B \log _{2}\left(1+S I N R^{1}\right) \leq 2^{\frac{\gamma}{2}} \frac{P_{\max }}{P_{\min }} B
$$

due to the fact that $\log _{2}(1+x)<x$ for $x>0$.

Similarly, at tier $j$, the minimum distance between a transmitter and a receiver, denoted by $d_{m i n}^{j}$, is

$$
d_{\text {min }}^{j}=\left(1+2+\ldots+2^{j-2}+1\right) \cdot l=2^{j-1} l,
$$

and

$$
P_{r}^{j} \leq \frac{P_{\max }}{\left(2^{j-1} l\right)^{\gamma}} .
$$

Denote by $d_{\max }^{j}$ the maximum distance between another transmitter in the same block and the receiver. Then,

$d_{\text {max }}^{j}=\sqrt{\left[2^{j-1} l+\left(2^{j-1}-1\right) l\right]^{2}+\left[\left(2^{j-1}-1\right) l\right]^{2} \times 2}$.
Let $n_{j}$ denote the number of nodes in a block at tier $j$. So we get

$$
\begin{aligned}
I^{j} & \geq n_{j} \frac{P_{\min }}{\left(d_{\max }^{j}\right)^{\gamma}} \\
& \geq \frac{n_{j} P_{\min }}{\left(\sqrt{\left(2^{j} l\right)^{2}+2\left(2^{j-1} l\right)^{2}}\right)^{\gamma}} \\
& =\frac{n_{j} P_{\min }}{\left(\sqrt{6} \cdot 2^{j-1} \cdot l\right)^{\gamma}} .
\end{aligned}
$$

As a result, we can obtain

$$
S I N R^{j} \leq \frac{6^{\frac{\gamma}{2}}}{n_{j}} \frac{P_{\max }}{P_{\min }}
$$

and hence

$$
\begin{aligned}
R_{C}^{j} & \leq n_{j} B \log _{2}\left(1+\frac{6^{\frac{\gamma}{2}}}{n_{j}} \frac{P_{\max }}{P_{\min }}\right) \\
& \leq 6^{\frac{\gamma}{2}} \frac{P_{\max }}{P_{\min }} B .
\end{aligned}
$$

We further denote the number of blocks at tier $j$ by $X_{j}$. Then, we have

$$
X_{j}=\frac{L^{2}}{l^{2}} \cdot\left(\frac{1}{4}\right)^{j-1} .
$$

Thus, the per-node throughput, i.e., $\lambda(n)$, satisfies

$$
\begin{aligned}
\lambda(n) & =\frac{\sum_{j} X_{j} R_{C}^{j}}{n / 2} \\
& \leq \frac{\frac{L^{2}}{l^{2}}\left(1+\frac{1}{4}+\frac{1}{16}+\ldots\right) 6^{\frac{\gamma}{2}} \frac{P_{\max }}{P_{\min }} B}{n / 2} \\
& =6^{\frac{\gamma}{2}} \frac{8 P_{\max }}{3 P_{\min }} B n^{-\frac{1}{3}} .
\end{aligned}
$$

We can thus have the following theorem.

Theorem 2: An upper bound on the capacity of 3D RANETs is

$$
\lambda(n)=\mathrm{O}\left(6^{\frac{\gamma}{2}} \frac{8 P_{\max }}{3 P_{\min }} B n^{-\frac{1}{3}}\right) .
$$

\section{CAPACITY OF THREE-DimENSIONAL HeTEROGENEOUS Ad Hoc NeTWORKS}

In this section, we explore the capacity of three-dimensional heterogeneous ad hoc networks (HANETs), where the distribution of the $n$ nodes follows a general NPP.

\section{A. A Lower Bound on Capacity}

We divide the network space into cubelets with an edge length of $l^{\prime}=\left(\frac{c^{\prime} \ln n}{\underline{\underline{ }}}\right)^{1 / 3}$, where $c^{\prime}\left(c^{\prime}>2\right)$ is a constant. Since $\underline{\Psi} n^{\alpha}=\omega(\ln n)$, we have $l^{\prime}=o\left(n^{\alpha / 3}\right)=o(L)$. Then, we have the following lemma.

Lemma 2: No cubelet is empty with high probability (w.h.p.).

Proof: For a cubelet $C_{b}$, the probability that there is no node in it, denoted by $P_{e}$, is

$$
P_{e}=e^{-\int_{C_{b}} \Psi(\xi) d \xi} \leq e^{-\underline{\Psi}\left(l^{\prime}\right)^{3}}=\frac{1}{n^{c^{\prime}}} .
$$




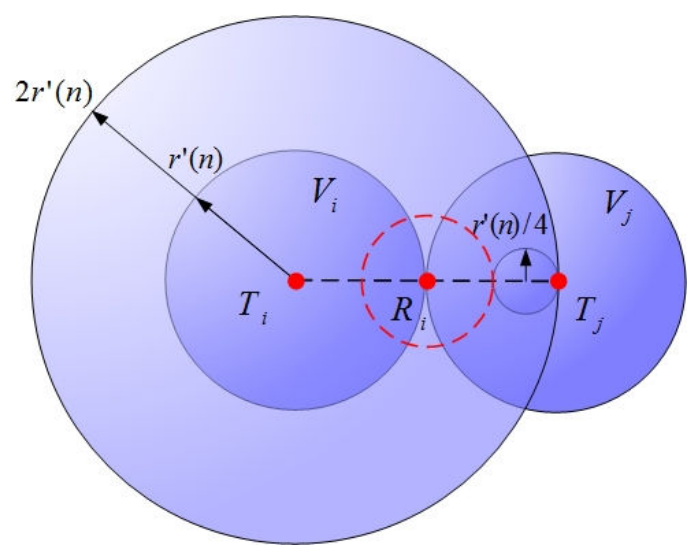

Fig. 4. Disjoint balls in a three-dimensional ad hoc networks.

So, $P_{e} \rightarrow 0$ as $n \rightarrow \infty$. Moreover, let $n_{c}$ be the number of cubelets in the network. We have $n_{c}=n^{\alpha} /\left(l^{\prime}\right)^{3}$. Then, the probability that at least one cubelet has no node in it, denoted by $P_{E}$, is

$$
P_{E} \leq n_{c} \cdot P_{e} \leq \frac{n^{\alpha}}{\left(l^{\prime}\right)^{3}} \cdot \frac{1}{n^{c^{\prime}}}=\frac{\underline{\Psi} n^{\alpha}}{\left(c^{\prime} \ln n\right) n^{c^{\prime}}} .
$$

Since $\underline{\Psi} n^{\alpha} \leq n$ and $c^{\prime}>2$, we can get that

$$
P_{E} \leq \frac{1}{\left(c^{\prime} \ln n\right) n^{c^{\prime}-1}} \rightarrow 0,
$$

i.e., no cubelet is empty w.h.p..

We let all nodes employ the same transmission power $P_{t}^{\prime}$ so that the transmission range is $r^{\prime}(n)=\sqrt{6} l^{\prime}$. Thus, we can enable the transmissions between any two nodes located in two neighboring cubelets. We also choose the physical carrier sensing range to be $2 r^{\prime}(n)$. Then, as shown in Fig. 4 , the balls centered at the transmitters transmitting at the same time with radius $r^{\prime}(n)$ are disjoint. So are the inside balls with radius $r^{\prime}(n) / 4$ and a transmitter on the boundary.

Consider a transmission from a transmitter $T_{i}$ to a receiver $R_{i}$ located at point $\xi_{0} \in \mathbb{R}^{3}$. The reception power level at $\xi_{0}$, denoted by $P_{r}\left(\xi_{0}\right)$, is

$$
P_{r}\left(\xi_{0}\right) \geq \frac{C P_{t}^{\prime}}{\left(r^{\prime}(n)\right)^{\gamma}} .
$$

Let $\mathbb{T}=\left\{x_{k}\right\}$ denote the set of transmitters transmitting at the same time as $T_{i}$, where $x_{k}$ also stands for the position of a node. We also denote the transmission volume by $\left|V_{t}\right|$. Then, the cumulative interference suffered at $\xi_{0}$, denoted by $I\left(\xi_{0}\right)$, is

$$
\begin{aligned}
I\left(\xi_{0}\right) & =\sum_{x_{k} \in \mathbb{T}} \frac{C P_{t}^{\prime}}{\left\|x_{k}-\xi_{0}\right\|^{\gamma}} \\
& =C P_{t}^{\prime} \sum_{x_{k} \in \mathbb{T}} \frac{1}{\left\|x_{k}-\xi_{0}\right\|^{\gamma}} \cdot \frac{1}{\left|V_{t}\right| / 64} \cdot\left(\left|V_{t}\right| / 64\right) \\
& \leq \frac{64 C P_{t}^{\prime}}{\left|V_{t}\right|} \int_{0}^{2 \pi} \int_{0}^{\pi} \int_{r^{\prime}(n) / 2}^{\sqrt{3} L} \frac{1}{\rho^{\gamma}} \cdot \rho^{2} \sin \phi \\
& =\frac{256 \pi C P_{t}^{\prime}}{\left|V_{t}\right|} \int_{r^{\prime}(n) / 2}^{\sqrt{3} L} \rho^{2-\gamma} \mathbf{d} \rho .
\end{aligned}
$$

Case I: $\gamma=2$.

When $\gamma=2$, the cumulative interference can be calculated as:

$$
\begin{aligned}
I\left(\xi_{0}\right) & \leq \frac{256 \pi C P_{t}^{\prime}}{\left|V_{t}\right|} \int_{r^{\prime}(n) / 2}^{\sqrt{3} L} 1 \mathbf{d} \rho \\
& \leq \frac{256 \pi C P_{t}^{\prime}}{\frac{4}{3} \pi\left(r^{\prime}(n)\right)^{3}} \cdot \sqrt{3} L \\
& =\frac{192 \sqrt{3} C P_{t}^{\prime} L}{\left(r^{\prime}(n)\right)^{3}} .
\end{aligned}
$$

As mentioned before, we consider an interference dominated environment. Thus, the SINR suffered by the receiver at $\xi_{0}$, denoted by $\operatorname{SINR}\left(\xi_{0}\right)$, is

$$
\operatorname{SINR}\left(\xi_{0}\right) \geq \frac{C P_{t}^{\prime} /\left(r^{\prime}(n)\right)^{2}}{192 \sqrt{3} C P_{t}^{\prime} L /\left(r^{\prime}(n)\right)^{3}} .
$$

Recall that $r^{\prime}(n)=\sqrt{6}\left(\frac{c^{\prime} \ln n}{\underline{\Psi}}\right)^{1 / 3}$ and $L=n^{\alpha / 3}$. We can get

$$
\operatorname{SINR}\left(\xi_{0}\right) \geq \frac{1}{96 \sqrt{2}} \cdot\left(\frac{c^{\prime} \ln n}{\underline{\Psi} n^{\alpha}}\right)^{\frac{1}{3}} .
$$

Thus, the transmission rate from $T_{i}$ to $R_{i}$, denoted by $R_{C}^{i}$, is

$$
R_{C}^{i} \geq B \log _{2}\left(1+\frac{1}{96 \sqrt{2}} \cdot\left(\frac{c^{\prime} \ln n}{\underline{\Psi} n^{\alpha}}\right)^{\frac{1}{3}}\right) .
$$

Let $c_{1}^{\prime}=\frac{1}{96 \sqrt{2}}$. Since $\underline{\Psi} n^{\alpha}=\omega(\ln n)$, then $\ln n /\left(\underline{\Psi} n^{\alpha}\right) \rightarrow$ 0 , and hence

$$
R_{C}^{i} \geq c_{1}^{\prime} B\left(\frac{c^{\prime} \ln n}{\underline{\Psi} n^{\alpha}}\right)^{\frac{1}{3}} .
$$

Case II: $2<\gamma<3$.

When $2<\gamma<3$, we can get

$$
\begin{aligned}
I\left(\xi_{0}\right) & \leq \frac{256 \pi C P_{t}^{\prime}}{\left|V_{t}\right|} \int_{r^{\prime}(n) / 2}^{\sqrt{3} L} \rho^{2-\gamma} \mathbf{d} \rho \\
& \leq \frac{256 \pi C P_{t}^{\prime}}{\frac{4}{3} \pi\left(r^{\prime}(n)\right)^{3}} \cdot \frac{(\sqrt{3} L)^{3-\gamma}}{3-\gamma} \\
& =\frac{192 C P_{t}^{\prime}(\sqrt{3} L)^{3-\gamma}}{(3-\gamma)\left(r^{\prime}(n)\right)^{3}}
\end{aligned}
$$

and hence

$$
\begin{aligned}
\operatorname{SINR}\left(\xi_{0}\right) & \geq \frac{C P_{t}^{\prime} /\left(r^{\prime}(n)\right)^{\gamma}}{\frac{192 C P_{t}^{\prime}(\sqrt{3} L)^{3-\gamma}}{(3-\gamma)\left(r^{\prime}(n)\right)^{3}}} \\
& =\frac{(3-\gamma) 2^{\frac{3-\gamma}{2}}}{192} \cdot\left(\frac{c^{\prime} \ln n}{\underline{\Psi} n^{\alpha}}\right)^{\frac{3-\gamma}{3}} .
\end{aligned}
$$

Thus, the transmission rate from $T_{i}$ to $R_{i}$, i.e., $R_{C}^{i}$, can be obtained by

$$
R_{C}^{i} \geq B \log _{2}\left(1+\frac{(3-\gamma) 2^{\frac{3-\gamma}{2}}}{192} \cdot\left(\frac{c^{\prime} \ln n}{\underline{\Psi} n^{\alpha}}\right)^{\frac{3-\gamma}{3}}\right) .
$$


Let $c_{2}^{\prime}=\frac{(3-\gamma) 2^{\frac{3-\gamma}{2}}}{192}$. Then we can get

$$
R_{C}^{i} \geq c_{2}^{\prime} B\left(\frac{c^{\prime} \ln n}{\underline{\Psi} n^{\alpha}}\right)^{\frac{3-\gamma}{3}} .
$$

Case III: $\gamma=3$.

When $\gamma=3$, we can obtain that

$$
\begin{aligned}
I\left(\xi_{0}\right) & \leq \frac{256 \pi C P_{t}^{\prime}}{\left|V_{t}\right|} \int_{r^{\prime}(n) / 2}^{\sqrt{3} L} \rho^{-1} \mathbf{d} \rho \\
& =\frac{256 \pi C P_{t}^{\prime}}{\frac{4}{3} \pi\left(r^{\prime}(n)\right)^{3}} \cdot\left[\ln \sqrt{3} L-\ln \frac{r^{\prime}(n)}{2}\right] \\
& =\frac{192 C P_{t}^{\prime}}{\left(r^{\prime}(n)\right)^{3}} \ln \frac{2 \sqrt{3} L}{r^{\prime}(n)},
\end{aligned}
$$

As a result, the SINR of the transmission from $T_{i}$ to $R_{i}$ located at $\xi_{0}$, is

$$
\begin{aligned}
\operatorname{SINR}\left(\xi_{0}\right) & \geq \frac{C P_{t}^{\prime} /\left(r^{\prime}(n)\right)^{3}}{\frac{192 C P_{t}^{\prime}}{\left(r^{\prime}(n)\right)^{3}} \ln \frac{2 \sqrt{3} L}{r^{\prime}(n)}} \\
& =\frac{1}{192 \ln \frac{2 \sqrt{3} n^{\alpha / 3}}{\sqrt{6}\left(\frac{c^{\prime} \ln n}{\frac{\Psi}{\Psi}}\right)^{1 / 3}}} \\
& =\frac{1}{192\left(\ln \sqrt{2}+\frac{1}{3} \ln \frac{\Psi n^{\alpha}}{c^{\prime} \ln n}\right)} \\
& \geq \frac{1}{256 \ln \frac{\underline{\Psi} n^{\alpha}}{c^{\prime} \ln n}} .
\end{aligned}
$$

The last step is due to the fact that $\ln \sqrt{2}<\ln \frac{\Psi n^{\alpha}}{c^{\prime} \ln n}$. Thus, the transmission rate from $T_{i}$ to $R_{i}$, i.e., $R_{C}^{i}$, can be calculated as

$$
R_{C}^{i} \geq B \log _{2}\left(1+\frac{1}{256 \ln \frac{\Psi n^{\alpha}}{c^{\prime} \ln n}}\right) \approx c_{3}^{\prime} B \ln ^{-1}\left(\frac{\underline{\Psi} n^{\alpha}}{c^{\prime} \ln n}\right) .
$$

where $c_{3}^{\prime}=1 / 256$.

Case IV: $\gamma>3$.

When $\gamma>3$, the cumulative interference can be calculated as

$$
\begin{aligned}
I\left(\xi_{0}\right) & \leq \frac{256 \pi C P_{t}^{\prime}}{\left|V_{t}\right|} \int_{r^{\prime}(n) / 2}^{\sqrt{3} L} \rho^{2-\gamma} \mathbf{d} \rho \\
& =\left.\frac{256 \pi C P_{t}^{\prime}}{\frac{4}{3} \pi\left(r^{\prime}(n)\right)^{3}} \cdot \frac{\rho^{3-\gamma}}{3-\gamma}\right|_{r^{\prime}(n) / 2} ^{\sqrt{3} L} \\
& \leq \frac{192 C P_{t}^{\prime}}{(\gamma-3) 2^{3-\gamma}}\left(r^{\prime}(n)\right)^{-\gamma} .
\end{aligned}
$$

Thus, the SINR of the transmission from $T_{i}$ to $R_{i}$, located at $\xi_{0}$, is

$$
\begin{aligned}
\operatorname{SINR}\left(\xi_{0}\right) & \geq \frac{C P_{t}^{\prime} /\left(r^{\prime}(n)\right)^{\gamma}}{\frac{192 C P_{t}^{\prime}}{(\gamma-3) 2^{3-\gamma}}\left(r^{\prime}(n)\right)^{-\gamma}} \\
& =\frac{(\gamma-3) 2^{3-\gamma}}{192},
\end{aligned}
$$

which can be lower bounded by a constant.

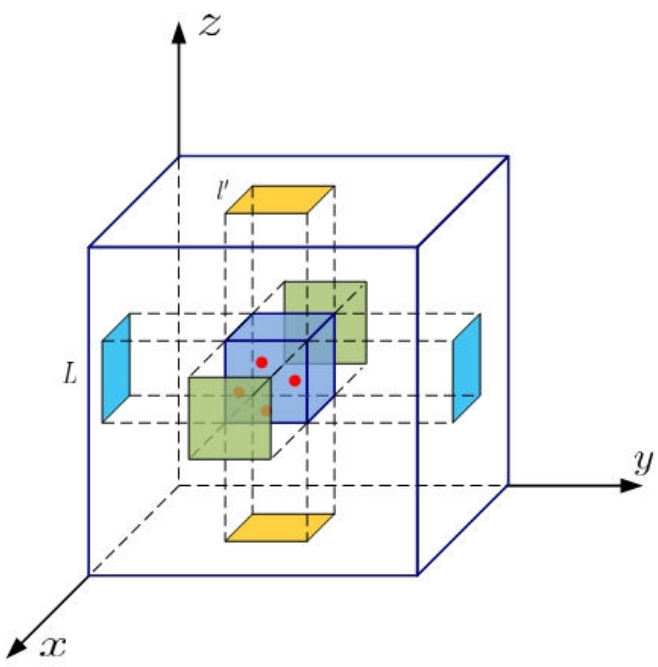

Fig. 5. The routing strategy used in nonhomogeneous three-dimensional ad hoc networks.

As a result, by letting $c_{4}^{\prime}=\frac{(\gamma-3) 2^{3-\gamma}}{192}$, the transmission rate from $T_{i}$ to $R_{i}$ is

$$
R_{C}^{i} \geq B \log _{2}\left(1+c_{4}^{\prime}\right) .
$$

Thus, we have the following lemma.

Lemma 3: In 3D HANETs, the data rate of each transmission, denoted by $R_{C}^{\prime}$, is:

$$
R_{C}^{\prime} \geq \begin{cases}c_{1}^{\prime} B\left(\frac{c^{\prime} \ln n}{\underline{\underline{\Psi} n^{\alpha}}}\right)^{\frac{1}{3}} & \text { if } \gamma=2, \\ c_{2}^{\prime} B\left(\frac{c^{\prime} \ln n}{\underline{\underline{m} n^{\alpha}}}\right)^{\frac{3-\gamma}{3}} & \text { if } 2<\gamma<3, \\ c_{3}^{\prime} B \ln ^{-1}\left(\frac{\underline{y} n^{\alpha}}{c^{\prime} \ln n}\right) & \text { if } \gamma=3, \\ B \log _{2}\left(1+c_{4}^{\prime}\right) & \text { if } \gamma>3 .\end{cases}
$$

Notice that nodes' transmission range $r^{\prime}(n)$ is chosen to be

$$
r^{\prime}(n)=\sqrt{6} l^{\prime}=\sqrt{6}\left(\frac{c^{\prime} \ln n}{\underline{\Psi}}\right)^{\frac{1}{3}} .
$$

So the maximum number of nodes that share the transmission rate $R_{C}^{\prime}$, denoted by $N_{n}$, is

$$
N_{n}=\bar{\Psi} \cdot 8\left|V_{t}\right|=\bar{\Psi} \cdot \frac{32}{3} \pi\left(r^{\prime}(n)\right)^{3}=64 \sqrt{6} \pi c^{\prime} \frac{\bar{\Psi} \ln n}{\underline{\Psi}} .
$$

Besides, we employ a routing strategy similar to that in Section III-A. As shown in Fig. 5, the maximum number of nodes that each cubelet relays packets for, denoted by $N_{r}$, can be obtained by $N_{r}=3\left(l^{\prime}\right)^{2} L \cdot \bar{\Psi}$. Since the minimum number of nodes in each cubelet, denoted by $n_{c}$, is $n_{c}=\underline{\Psi}\left(l^{\prime}\right)^{3}$, the maximum average traffic load for each node, denoted by $\bar{H}^{\prime}$, is

$$
\bar{H}^{\prime}=\frac{N_{r}}{n_{c}}=\frac{3 L \bar{\Psi}}{\underline{\Psi} l^{\prime}}=\frac{3 \bar{\Psi}}{\underline{\Psi}}\left(\frac{\underline{\Psi} n^{\alpha}}{c^{\prime} \ln n}\right)^{\frac{1}{3}} .
$$

As a result, a per-node throughput, denoted by $\lambda^{\prime}(n)$, is feasible if the following holds:

$$
\lambda^{\prime}(n) \bar{H}^{\prime} \leq \frac{R_{C}^{\prime}}{N_{n}},
$$


i.e.,

$$
\lambda^{\prime}(n) \leq \frac{1}{192 \sqrt{6} \pi c^{\prime}} \cdot \frac{\underline{\Psi}^{2}}{\bar{\Psi}^{2} \ln n} \cdot\left(\frac{c^{\prime} \ln n}{\underline{\Psi} n^{\alpha}}\right)^{\frac{1}{3}} \cdot R_{C}^{\prime} .
$$

We can thus have the following theorem.

Theorem 3: A lower bound on the capacity of 3D HANETs is

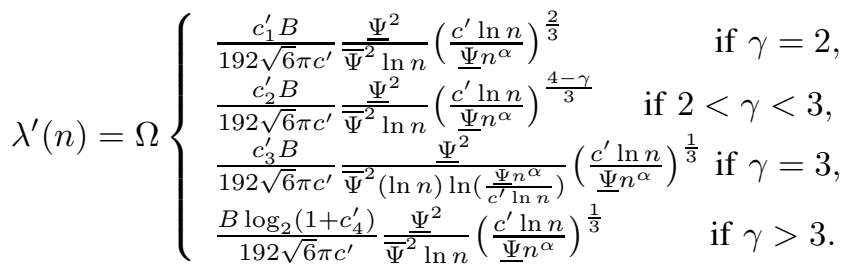

\section{B. An Upper Bound on Capacity}

We then use percolation theory [9], [26] to find an upper bound on the capacity of 3D heterogeneous ad hoc networks.

We divide the network space into cubelets with an edge length of $l_{c}^{\prime}$. Then, the 3D network space can be decomposed into $L / l_{c}^{\prime}$ 2D planar areas each of which contains $\left(L / l_{c}^{\prime}\right) \times$ $\left(L / l_{c}^{\prime}\right)$ cubelets. Thus, the capacity of the 3D network can be upper bounded by the sum of the capacity of each individual 2D planar network with sources and destinations on the same plane.

Let $0<p<1$ be a constant. Choosing

$$
\left(l_{c}^{\prime}\right)^{3}=-\frac{\ln p}{\bar{\Psi}},
$$

we know that the probability that a cubelet $C_{b}$ is empty, denoted by $P_{e}$, is

$$
P_{e}=e^{-\int_{C_{b}} \Psi(\xi) d \xi} \geq e^{-\bar{\Psi}\left(l_{c}^{\prime}\right)^{3}}=p .
$$

Since $p_{c} \approx 0.59$ is the critical probability of independent site percolation in a square lattice, choosing $p>p_{c}$ can make all $L / l_{c}^{\prime} 2 \mathrm{D}$ planar areas percolated. Moreover, in each 2D planar network, inside any rectangle of size $L \times(\kappa \log n) l_{c}^{\prime}$ $(\kappa>0)$, there exists at least one path composed of $\Theta\left(L / l_{c}^{\prime}\right)$ empty cublets connecting the top side with the bottom side of the network.

Consider one of the 2D planar networks as shown in Fig. 6 . We choose a rectangle to the right of $y=y_{0}$ with width $h=(\kappa \log n) l_{c}^{\prime}$ so that on the left side of $y=y_{0}$ there are $n / 2$ nodes in the $3 \mathrm{D}$ cube. Then, inside this rectangle there is at least one crossing path composed of $\Theta\left(L / l_{c}^{\prime}\right)$ empty cubelets. Our objective is to find an upper bound on the amount of information $\mathcal{I}$ that can traverse from left to right through the cut planes in the 3D network. Since there are at least $n / 2$ endto-end data flows going through the cut planes, the per-node throughput can be upper bounded by $\mathcal{I} /(n / 2)$.

We further divide the left part of the cutting plane (as shown in Fig. 6) into tiers of blocks. Blocks at different tiers are of different sizes. Specifically, the tier $j(j \geq 1)$ blocks are of size $\left(2^{j-1} l_{c}^{\prime}\right) \times\left(2^{j-1} l_{c}^{\prime}\right) \times l_{c}^{\prime}$. Besides, Tier 1 blocks are those right next to the rightmost empty cubelets along the crossing path (as shown in Fig. 6). Tier 2 blocks are next to Tier 1 blocks, and so on and so forth.

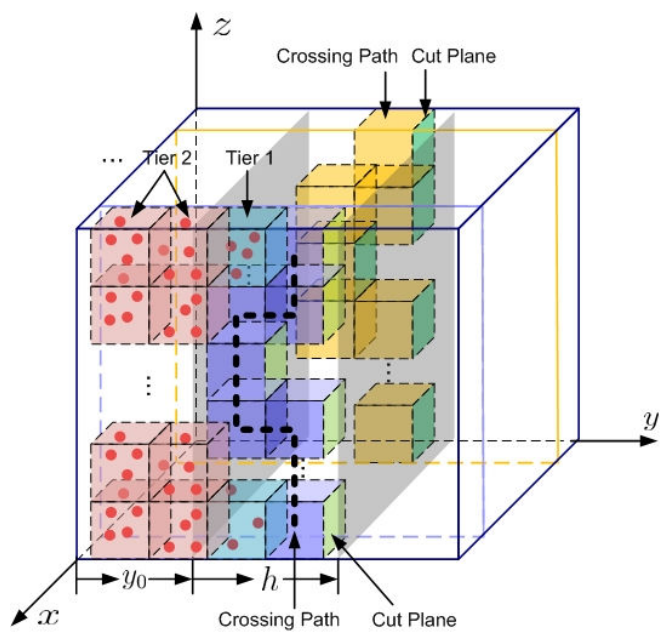

Fig. 6. Finding an upper bound by decomposing a 3D network into 2D networks.

As a result, the minimum distance between a transmitter in a tier $j$ block and its corresponding receiver, denoted by $d_{\text {min }}^{j}$, is

$$
d_{\text {min }}^{j}=\left(1+1+2+\ldots+2^{j-2}\right) \cdot l_{c}^{\prime}=2^{j-1} l_{c}^{\prime},
$$

and since the transmission power of the nodes ranges from $P_{\min }$ to $P_{\max }$, the reception power at the receiver, denoted by $P_{r}^{j}$, is

$$
P_{r}^{j} \leq \frac{C P_{\max }}{\left(2^{j-1} l_{c}^{\prime}\right)^{\gamma}} .
$$

Denote by $d_{\max }^{j}$ the maximum distance between another transmitter in the same block and the receiver. Then,

$$
d_{\text {max }}^{j}=\sqrt{\left(2^{j-1}+2^{j-1}\right)^{2}+\left(2^{j-1}\right)^{2}+1} \cdot l_{c}^{\prime} .
$$

Let $n_{j}$ denote the number of nodes in a block at tier $j$. The cumulative interference suffered at the receiver, denoted by $I^{j}$, can be obtained as

$$
I^{j} \geq n_{j} \frac{C P_{\min }}{\left(d_{\max }^{j}\right)^{\gamma}} \geq \frac{n_{j} C P_{\min }}{\left(\sqrt{3} \cdot 2^{j} l_{c}^{\prime}\right)^{\gamma}} .
$$

As a result, we can get

$$
S I N R^{j} \leq \frac{(2 \sqrt{3})^{\gamma}}{n_{j}} \frac{P_{\max }}{P_{\min }}
$$

and hence

$$
\begin{aligned}
R_{C}^{j} & \leq n_{j} B \log _{2}\left(1+\frac{(2 \sqrt{3})^{\gamma}}{n_{j}} \frac{P_{\max }}{P_{\min }}\right) \\
& \leq(2 \sqrt{3})^{\gamma} \frac{P_{\text {max }}}{P_{\text {min }}} B .
\end{aligned}
$$

We denote the number of non-empty tier $j$ blocks in each 2D planar network by $Y_{j}$. Since as shown in Fig. 6 the crossing path might go through some of the blocks, some blocks may be empty. Thus, we have

$$
Y_{j} \leq \frac{L}{l_{c}^{\prime}} \cdot\left(\frac{1}{2}\right)^{j-1} .
$$


So, the per-node throughput, i.e., $\lambda^{\prime}(n)$, satisfies

$$
\begin{aligned}
\lambda^{\prime}(n) & =\frac{\left(L / l_{c}^{\prime}\right) \cdot \sum_{j} Y_{j} R_{C}^{j}}{n / 2} \\
& \leq \frac{\frac{L^{2}}{\left(l_{c}^{\prime}\right)^{2}}\left(1+\frac{1}{2}+\frac{1}{4}+\ldots\right)(2 \sqrt{3})^{\gamma} \frac{P_{\max }}{P_{\min }} B}{n / 2} \\
& =(2 \sqrt{3})^{\gamma} \frac{4 P_{\max }}{P_{\min }} B\left(\frac{\bar{\Psi}^{2} n^{2 \alpha-3}}{\ln ^{2} p}\right)^{\frac{1}{3}} .
\end{aligned}
$$

Moreover, since in the network there are at most $n$ non-empty blocks, we also have

$$
\lambda^{\prime}(n) \leq \frac{n \cdot R_{C}^{j}}{n / 2} \leq(2 \sqrt{3})^{\gamma} \frac{2 P_{\max }}{P_{\min }} B
$$

We can thus have the following theorem.

Theorem 4: An upper bound on the capacity of 3D HANETs is

$$
\begin{aligned}
\lambda^{\prime}(n)= & \mathrm{O}\left(\operatorname { m i n } \left\{(2 \sqrt{3})^{\gamma} \frac{2 P_{\max }}{P_{\min }} B,\right.\right. \\
& \left.\left.(2 \sqrt{3})^{\gamma} \frac{2 P_{\max }}{P_{\min }} B\left(\frac{\bar{\Psi}^{2} n^{2 \alpha-3}}{\ln ^{2} p}\right)^{\frac{1}{3}}\right\}\right) .
\end{aligned}
$$

Considering a special case when $\bar{\Psi}=\Theta(\underline{\Psi})$, we get $\bar{\Psi} n^{\alpha}=$ $\underline{\Psi} n^{\alpha}=n$. Therefore, we can have $\lambda^{\prime}(n)=\mathrm{O}\left(n^{-\frac{1}{3}}\right)$.

\section{CONCLUSION}

In this paper, we have explored the capacity of threedimensional wireless ad hoc networks, including 3D regular ad hoc networks (RANETs) and 3D heterogeneous ad hoc networks (HANETs). Both lower and upper bounds have been obtained for the two types of networks when the path loss exponent is no less than 2. We find that lower bounds in the networks are dependent on the power propagation environment, i.e., the path loss exponent, while upper bounds are not. In our future work, we will investigate how to further bridge the gap between the lower bounds and the upper bounds on the capacity of 3D ad hoc networks.

\section{REFERENCES}

[1] S. Aeron and V. Saligrama. Wireless ad hoc networks: Strategies and scaling laws for the fixed snr regime. IEEE Transactions on Information Theory, 53(6):2044-2059, June 2007.

[2] G. Alfano, M. Garetto, and E. Leonardi. Capacity scaling of wireless networks with inhomogeneous node density: Upper bounds. IEEE Journal on Selected Areas in Communications (JSAC) - Special Issue on Stochastic Geometry and Random Graphs for the Analysis and Design of Wireless Networks, 27(7):1147-1157, September 2009.

[3] N. Bansal and Z. Liu. Capacity, delay, and mobility in wireless adhoc networks. In Proceeding of the IEEE International Conference on Computer Communications (INFOCOM'03), San Francisco, California, USA, March 2003.

[4] C. Buraagohain, S. Suri, C. Toth, and Y. Zhou. Improved throughput bounds for interference-aware routing in wireless networks. In Proceeding of COCOON, Banff, Alberta, Canada, July 2007.

[5] B. Burgstaller and F. Pillichshammer. The average distance between two points. Bulletin of the Australian Mathematical Society, 80:353359,2009

[6] O. Dousse, M. Franceschetti, and P. Thiran. On the throughput scaling of wireless relay networks. IEEE Transactions on Information Theory (Joint special issue with IEEE/ACM Transactions on Networking), 52(6):2756-2761, 2006.
[7] E. Duarte-Melo, A. Josan, M. Liu, D. Neuhoff, and S. Pradhan. The effect of node density and propagation model on throughput scaling of wireless networks. In Proceedings of IEEE ISIT, Seatlle, Washington, USA, July 2006.

[8] M. Franceschetti, O. Dousse, D. N. Tse, and P. Thiran. Closing the gap in the capacity of wireless networks via percolation theory. IEEE Transactions on Information Theory, 53(3):1009-1018, 2007.

[9] G. Grimmett. Percolation (Second Edition). Springer-Verlag Berlin Heidelberg, New York, 1999.

[10] M. Grossglauser and D. Tse. Mobility increases the capacity of ad hoc wireless networks. IEEE/ACM Transactions on Networking, 10(4):477486, August 2002.

[11] P. Gupta and P. Kumar. The capacity of wireless networks. IEEE Transactions on Information Theory, 46(2):388-404, March 2000.

[12] P. Gupta and P. Kumar. Internets in the sky: The capacity of three dimensional wireless networks. Communications in Information and Systems, 1:33-50, 2001.

[13] C. Hu, X. Wang, and F. Wu. Motioncast: On the capacity and delay tradeoffs. In Proceeding of ACM MobiHoc, New Orleans, Louisiana, USA, May 2009.

[14] L. Law, S. Krishnamurthy, and M. Faloutsos. Capacity of hybrid cellular-ad hoc data networks. In Proceeding of the IEEE International Conference on Computer Communications (INFOCOM'08), Phoenix, Arizona, USA, April 2008.

[15] P. Li and Y. Fang. Impacts of topology and traffic pattern on capacity of hybrid wireless networks. IEEE Transactions on Mobile Computing, 8(12):1585-1595, December 2009.

[16] P. Li and Y. Fang. The capacity of heterogeneous wireless networks. In Proceeding of IEEE INFOCOM, San Diego, California, USA, March 2010.

[17] P. Li, Y. Fang, and J. Li. Throughput, delay, and mobility in wireless ad hoc networks. In Proceeding of IEEE INFOCOM, San Diego, California, USA, March 2010.

[18] P. Li, X. Huang, and Y. Fang. Capacity scaling of multihop cellular networks. In Proceeding of IEEE INFOCOM, Shanghai, China, April 2011.

[19] P. Li, C. Zhang, and Y. Fang. Capacity and delay of hybrid wireless broadband access networks. IEEE Journal on Selected Areas in Communications (JSAC) - Special Issue on Broadband Access Networks, 27(2):117-125, February 2009.

[20] X. Lin, G. Sharma, R. Mazumdar, and N. Shroff. Degenerate delay-capacity tradeoffs in ad-hoc networks with brownian mobility. IEEE/ACM Transactions on Networking, Special Issue on Networking and Information Theory, 14:2777-2784, 2006.

[21] B. Liu, Z. Liu, and D. Towsley. On the capacity of hybrid wireless networks. In Proceeding of the IEEE International Conference on Computer Communications (INFOCOM'03), San Francisco, California, USA, March 2003.

[22] A. Ozgur, O. Leveque, and D. Tse. How does the information capacity of ad hoc networks scale? In Proceedings of the Forty-fourth Annual Allerton Conference on Communication, Control and Computing, Monticello, IL, USA, September 2006.

[23] A. Ozgur, O. Leveque, and D. Tse. Hierarchical cooperation achieves optimal capacity scaling in ad hoc networks. IEEE Transactions on Information Theory, 53(10):3549-3572, October 2007.

[24] C. Peraki and S. Servetto. On the maximum stable throughput problems in random networks with directional antennas. In Proceedings of ACM MobiHoc, Annapolis, Maryland, USA, June 2003.

[25] T. Rappaport. Wireless Communications: Principles and Practice (Second Edition). Prentice-Hall PTR, 2002.

[26] D. Stauffer and A. Aharony. Introduction to Percolation Theory (Second Edition). CRC Press, 1994.

[27] S. Toumpis. Capacity bounds for three classes of wireless networks. In Proceeding of ACM MobiHoc, Roppongi Hills, Tokyo, Japan, May 2004.

[28] X. Wang, Y. Bei, Q. Peng, and L. Fu. Speed improves delay-capacity tradeoff in motioncast. IEEE Transactions on Parallel and Distributed Systems, 1(99):1-13, 2010.

[29] A. Zemlianov and G. Veciana. Capacity of ad hoc wireless networks with infrastructure support. IEEE Journal on Selected Areas in Communications, 23(3), March 2005.

[30] G. Zhang, Y. Xu, X. Wang, and M. Guizani. Capacity of hybrid wireless networks with directional antenna and delay constraint. IEEE Transactions on Communications, 58(7):2097-2106, 2010. 